\title{
SEOM clinical guidelines for the diagnosis and treatment of gastroenteropancreatic neuroendocrine neoplasms (GEP-NENs) 2014
}

\author{
R. Garcia-Carbonero $\cdot$ P. JImenez-Fonseca $\cdot$ \\ A. Teulé $\cdot$ J. Barriuso $\cdot$ I. Sevilla
}

Received: 5 August 2014/ Accepted: 6 August 2014/Published online: 3 September 2014

(c) Federación de Sociedades Españolas de Oncología (FESEO) 2014

\begin{abstract}
GEP-NENs are a challenging family of tumors of growing incidence and varied clinical management and behavior. Diagnostic techniques have substantially improved over the past decades and significant advances have been achieved in the understanding of the molecular pathways governing tumor initiation and progression. This has already translated into relevant advances in the clinic. This guideline aims to provide practical recommendations for the diagnosis and treatment of GEP-NENs. Diagnostic
\end{abstract}

R. Garcia-Carbonero $(\square)$

Medical Oncology Department, Hospital Universitario Virgen del Rocio, Instituto de Biomedicina de Sevilla (IBIS)

(Universidad de Sevilla, CSIC, HUVR), Center affiliated to the

Red Temática de Investigación Cooperativa en Cancer (RTICC), Instituto Carlos III, Spanish Ministry of Science and Innovation, Av. Manuel Siurot, s/n, 41013 Seville, Spain

e-mail:rgcarbonero@gmail.com

P. JImenez-Fonseca

Medical Oncology Department, Hospital Universitario Central de Asturias, Asturias, Spain

\section{A. Teulé}

Medical Oncology Department, Instituto Catalán de Oncología (ICO), Barcelona, Spain, Center affiliated to the Red Temática de Investigación Cooperativa en Cancer (RTICC), Instituto Carlos III, Spanish Ministry of Science and Innovation, Seville, Spain

\section{J. Barriuso}

Faculty of Life Sciences, University of Manchester, Manchester, UK

\section{J. Barriuso}

Department of Medical Oncology, The Christie NHS Foundation Trust, Manchester, UK

I. Sevilla

Medical Oncology Department, Hospital Universitario Virgen de la Victoria y Hospital Regional Universitario, Málaga, Spain workup, histological and staging classifications, and the different available therapeutic approaches, including surgery, liver-directed ablative therapies, peptide receptor radionuclide therapy, and systemic hormonal, cytotoxic or targeted therapy, are briefly discussed in this manuscript. Clinical presentation (performance status, comorbidities, tumor-derived symptoms and hormone syndrome in functioning tumors), histological features [tumor differentiation, proliferation rate (Ki-67), and expression of somatostatin receptors], disease localization and extent, and resectability of primary and metastatic disease, are all key issues that shall be taken into consideration to appropriately tailor therapeutic strategies and surveillance of these patients.

Keywords Guidelines - Neuroendocrine tumors . Neuroendocrine neoplasms · Enteropancreatic · Diagnosis · Therapy

\section{Introduction}

The incidence of NENs in the Caucasian population has substantially increased over the past three decades from 1.09 (1973) to 5.76 (2007) new cases per 100,000 inhabitants annually. About two-thirds of NENs are of gastrointestinal or pancreatic origin (GEP-NENs), and, among these, the most common site of origin is the small intestine [1-4]. Although GEP-NENs represent only $2 \%$ of all gastrointestinal tumors, their prevalence is rather high due to their relatively long survival, being the second most prevalent gastrointestinal malignancy after colorectal adenocarcinoma.

NENs are diagnosed at a younger age than carcinomas, usually during the fifth decade of life, and they may arise 
sporadically or as a result of hereditary predisposition syndromes such as multiple endocrine neoplasia type 1 (MEN-1) or Von Hippel-Lindau's disease (VHL). Clinical onset in patients with genetic predisposition may occur 15 years earlier. About one-third are so-called functioning tumors, a unique feature that is the consequence of excess hormone production by the tumor. The rate of metastatic disease at presentation widely ranges in different tumor registries from 27 to $73 \%$ [1-4]. Overall survival rates may also significantly vary from 30 to $90 \%$ at 5 years, depending upon the distribution of a number of prognostic factors, the most relevant being the primary tumor site, stage of disease, histological differentiation and proliferative index (Ki-67 or mitotic index). A pancreatic origin, followed by the colon, is the primary tumor site with the worst prognosis among GEP-NETs [1].

The wide range of histological, biochemical and clinical behavior of these tumors may require very diverse diagnostic and therapeutic approaches, including surgery, liverdirected ablative techniques, peptide receptor radionuclide therapy (PRRT), and/or systemic hormonal, cytotoxic or targeted therapies. The aim of the current manuscript is to provide synthetic practical guidelines regarding diagnostic procedures and therapeutic options for the multidisciplinary management of NENs of gastrointestinal or pancreatic origin.

\section{Diagnostic procedures}

NENs originate in a great diversity of tissues and are characterized by their ability to produce different peptides that cause distinct hormonal syndromes (Table 1). Based on this, they are broadly subdivided into "functional" or "nonfunctional" tumors (with or without a clinical syndrome). Most well-differentiated neuroendocrine tumors (NETs) arise from the gut or bronchopulmonary system, formerly called carcinoid tumors. Current nomenclature guidelines do not support this term, to avoid confusion between tumor type and the functional syndrome. Carcinoid is also a generic term for a characteristic syndrome that results from the intermittent release of bioactive amines (serotonin and others) into the systemic circulation that occurs in some patients with NETs.

The most frequent functionally active neuroendocrine tumors are, besides carcinoids, insulinoma, gastrinoma, vasoactive intestinal polypeptidoma (VIPoma) and glucagonoma. Non-functional tumors are often clinically silent until late advanced disease. Although these neoplasms are generally more indolent than carcinomas, they often have unpredictable biological behavior and may sometimes present very aggressive clinical course. Given the rarity, heterogeneous and unique nature of these neoplasms, it is highly recommended to refer these patients to specialized centres.

In order to appropriately diagnose and stage GEP-NENs the following procedures are recommended:

- Medical history and physical examination.

- Laboratory tests, including hematological, liver and renal function tests.

- General hormonal markers: chromogranin A (NETs), neurospecific enolase (NECs).

- Specific hormonal markers: urinary 5-hydroxyindoleacetic acid (5-HIAA) (carcinoid syndrome); gastrin \pm secretin test (gastrinomas); insulin/glucose ratio, proinsulin, $\mathrm{C}$ peptide (insulinomas), glucagon, VIP and others depending upon clinical symptoms.

- Tumor specimen or biopsy: the histopathological report shall provide the WHO classification and TNM staging [5-8]. Inmunohistochemical staining should always include Ki-67 (\% of positive cells assessed in 2,000 tumor cells in areas of highest nuclear labeling) and general neuroendocrine markers (chromogranin A, synaptophysin and neuron-specific enolase) [9]. Specific markers are not mandatory and should only be

Table 1 Clinical features associated with excess hormone production in GEP-NETs

\begin{tabular}{|c|c|c|c|}
\hline Tumor & Hormone & $\begin{array}{l}\text { Islet cell } \\
\text { type }\end{array}$ & Clinical syndrome \\
\hline Carcinoid & Serotonin & & Flushing, diarrhea, bronchospasm, tricuspid or pulmonary valve insufficiency or stenosis \\
\hline Gastrinoma & Gastrin & $\gamma$ & Zollinger-Ellison syndrome: recurrent peptic ulcer, diarrhea/steatorrhea \\
\hline Insulinoma & Insulin & $\beta$ & Hypoglycemia, catecholamine excess \\
\hline Glucagonoma & Glucagon & $\alpha$ & $\begin{array}{l}\text { Diabetes mellitus, migratory necrolytic erythema, panhypoaminoaciduria, thromboembolism, } \\
\text { weight loss }\end{array}$ \\
\hline VIPoma & VIP & $\delta$ & $\begin{array}{l}\text { Verner-Morrison syndrome (WDHA): watery diarrhea, hypokalemia, achlorhydria, metabolic } \\
\text { acidosis, hyperglycemia, hypercalcemia, flushing }\end{array}$ \\
\hline Somatostatinoma & Somatostatin & $\delta$ & Diabetes mellitus, diarrhea/steatorrhea, hypochlorhydria, weight loss, gall bladder disease \\
\hline PPoma & $\mathrm{PP}$ & PP cells & Hepatomegaly, abdominal pain, occasional watery diarrhea \\
\hline PTHoma & PTH-rp & & Hypercalcemia \\
\hline
\end{tabular}


performed if clinically indicated (insulin, glucagon, etc).

- Somatostatin receptor scintigraphy (octreoscan). This may be replaced by new diagnostic techniques with improved resolution, such as ${ }^{68}$ Gallium-DOTA-TOC/NOC/-TATE positron emission tomography (PET), wherever available.

- Dynamic CT scan of the abdomen.

- Chest X-ray. A thoracic CT scan may be considered in poorly differentiated tumors, colon primaries or those in whom surgery of liver metastasis is being considered.

- Genetic counseling in hereditary predisposition syndromes (MEN-1, VHL, tuberous sclerosis, and neurofibromatosis, among others).

Depending upon clinical presentation and primary tumor site the following procedures may be also considered:

- Oral endoscopy, enteroscopy, capsule endoscopy, endoscopic ultrasound, colonoscopy.

- 18-Fluorodeoxyglucose-(FDG-) PET (poorly differentiated NEC metastasis of unknown primary; no use in low grade tumors).

- Echocardiogram (carcinoid syndrome).

- N-terminal pro-brain natriuretic peptide (NT-pro-BNP) may be also considered if carcinoid heart disease is suspected.

- Brain CT or bone scan (only if bone pain, neurological symptoms, etc).

\section{Classification and staging systems}

Recent international efforts are helping to improve the prognostic classifications of this type of tumors and to better tailor therapeutic strategies in these patients. In this context, the recently updated 2010 WHO classification has been a major step forward as a worldwide expert consensus for a standardized taxonomy of this heterogeneous disease [5]. The WHO classification has endorsed the European Neuroendocrine Tumor Society (ENETS) grading system, and discerns two major NEN pathological categories with important therapeutic and prognostic implications: welldifferentiated neuroendocrine tumors (NETs G1 and G2) and poorly differentiated neuroendocrine carcinomas (NECs G3) (Table 2). Less worldwide consensus exists regarding staging of these neoplasms. Indeed, two TNM staging systems have been proposed (ENETS and AJCC/ UICC) [6-8], that have relevant differences in certain subgroups of NENs including those of pancreatic or appendiceal origin (Table 3). In addition, the ENETS proposal stages poorly differentiated NECs as well-
Table 22010 WHO classification and grading of neuroendocrine neoplasms (NENs) of the digestive system

\begin{tabular}{llll}
\hline & Grade & $\begin{array}{l}\text { Mitotic count } \\
(10 \mathrm{HPF})\end{array}$ & $\begin{array}{l}\text { Ki 67 } \\
\text { index } \\
(\%)^{\mathrm{a}}\end{array}$ \\
\hline $\begin{array}{l}\text { Well-differentiated } \\
\text { neoplasms }\end{array}$ & $\begin{array}{l}\text { NET G1 } \\
\text { NET G2 }\end{array}$ & $<2$ & $\leq 2$ \\
$\begin{array}{l}\text { Poorly } \\
\text { differentiated } \\
\text { neoplasms }\end{array}$ & $\begin{array}{c}\text { NEC G3 (large- } \\
\text { or small-cell) }\end{array}$ & $>20$ & $3-20$ \\
\hline
\end{tabular}

NEC neuroendocrine carcinoma, NET neuroendocrine tumor, $W H O$ World Health Organization, $H P F$ high-power field $=2 \mathrm{~mm}^{2}$ (at least 40 fieldsat $40 \times$ magnification evaluated in areas of highest mitotic density)

${ }^{\text {a }}$ MIB1 antibody (\% of 2,000 tumor cells in areas of highest nuclear labeling). If Ki-67 index and mitotic index are inconsistent, choose the highest grade

differentiated NETs, while the AJCC system stages poorly differentiated NECs as adenocarcinomas. The unification of these staging systems is therefore a relevant pending issue that will likely require solid multi-institutional data for adequate and consistent validation.

\section{Therapy}

\section{Surgery}

Surgery is the only potentially curative therapeutic strategy in localized disease. Radical oncological surgery is indicated except for small carcinoids $(<2 \mathrm{~cm})$ of the stomach, appendix or rectum, in which more conservative surgical or endoscopic resections may be appropriate due to their low malignant potential. Small pancreatic insulinomas also have a very good prognosis (90\% are benign tumors) and tumor enucleation is generally sufficient $[3,4]$. No adjuvant therapy is recommended in completely resected welldifferentiated localized NETs. Adjuvant chemotherapy with platinum and etoposide may be considered in poorly differentiated tumors, although evidence supporting this strategy is lacking.

Surgery also plays a major role in advanced disease. Surgery of metastasic disease is recommended in G1-2 well-differentiated NETs if complete resection is deemed feasible. If complete resection of metastatic disease is performed no adjuvant treatment is recommended, but a randomized trial addressing this question is being initiated. Major cytoreductive therapy with palliative purposes may be considered even if $\mathrm{R} 0$ is not achievable in patients with extensive liver metastasis and hormonal syndrome refractory to medical therapy. Some advocate that this procedure may also extend survival and could therefore be considered 
Table 3 TNM classification (AJCC vs. ENETS)

\begin{tabular}{|c|c|}
\hline AJCC TNM (7th edition) & TNM proposed by ENETS \\
\hline Pancreas & Pancreas \\
\hline Tx: Primary tumor cannot be assessed & Tx: Primary tumor cannot be assessed \\
\hline T0: No evidence of primary tumor & T0: No evidence of primary tumor \\
\hline Tis: Carcinoma in situ & T1: Tumor confined to pancreas $<2 \mathrm{~cm}$ \\
\hline T1: Tumor confined to pancreas $\leq 2 \mathrm{~cm}$ & T2: Tumor confined to pancreas $2-4 \mathrm{~cm}$ \\
\hline T2: Tumor confined to pancreas $>2 \mathrm{~cm}$ & T3: Tumor confined to pancreas $>4 \mathrm{~cm}$ or invading duodenum or bile duct \\
\hline $\begin{array}{l}\text { T3: Tumor not confined to pancreas, but not involving coeliac trunk or } \\
\text { SMA }\end{array}$ & $\begin{array}{l}\text { T4: Tumor invading adjacent organs (stomach, spleen, colon, adrenal gland) or wall of large } \\
\text { vessels (coeliac trunk or SMA) }\end{array}$ \\
\hline \multicolumn{2}{|l|}{ T4: Tumor invading coeliac trunk or SMA } \\
\hline Appendix & Appendix \\
\hline Tx: Primary tumor cannot be assessed & Tx: Primary tumor cannot be assessed \\
\hline T0: No evidence of primary tumor & T0: No evidence of primary tumor \\
\hline $\mathrm{T} 1:$ Tumor $\leq 2 \mathrm{~cm}$ & T1: Tumor $\leq 1 \mathrm{~cm}$ invading submucosa and muscularis propria \\
\hline $\begin{array}{l}\text { T2: Tumor }>2-4 \mathrm{~cm} \text {; caecum } \\
\text { T3: Tumor }>4 \mathrm{~cm} \text {; ileum }\end{array}$ & $\begin{array}{l}\text { T2: Tumor } \leq 2 \mathrm{~cm} \text { invading submucosa and muscularis propria and/or minimally }(\leq 3 \mathrm{~mm} \text { ) } \\
\text { invading subserosa/mesoappendix }\end{array}$ \\
\hline T4: Tumor perforating peritoneum or invading other organs or structures & T3: Tumor $>2 \mathrm{~cm}$ and/or invading subserosa/mesoappendix $>3 \mathrm{~mm}$ \\
\hline & T4: Tumor invading peritoneum or other organs \\
\hline Stomach & Stomach \\
\hline Tx: Primary tumor cannot be assessed & Tx: Primary tumor cannot be assessed \\
\hline T0: No evidence of primary tumor & T0: No evidence of primary tumor \\
\hline Tis: $<0.5 \mathrm{~mm}$ confined to mucosa & Tis: Tumor in situ/dysplasia $(<0.5 \mathrm{~mm})$ \\
\hline $\mathrm{T} 1$ : Tumor $\leq 1 \mathrm{~cm}$ invading lamina propria or submucosa & $\mathrm{T} 1$ : Tumor $\leq 1 \mathrm{~cm}$ invading lamina propria or submucosa \\
\hline $\mathrm{T} 2$ : Tumor $>1 \mathrm{~cm}$ or invading muscularis propria & $\mathrm{T} 2$ : Tumor $>1 \mathrm{~cm}$ or invading muscularispropria or subserosa \\
\hline T3: Tumor invading subserosa & T3: Tumor penetrating serosa \\
\hline T4: Tumor perforating serosa or invading adjacent structures & T4: Tumor invading adjacent structures \\
\hline Small intestine & Small intestine \\
\hline Tx: Primary tumor cannot be assessed & Tx: Primary tumor cannot be assessed \\
\hline T0: No evidence of primary tumor & T0: No evidence of primary tumor \\
\hline T1: Tumor $\leq 1 \mathrm{~cm}$ invading lamina propia or submucosa & $\mathrm{T} 1$ : Tumor $\leq 1 \mathrm{~cm}$ invading lamina propia or submucosa \\
\hline $\mathrm{T} 2$ : Tumor $>1 \mathrm{~cm}$ or invading muscularis propia & $\mathrm{T} 2$ : Tumor $>1 \mathrm{~cm}$ or invading muscularispropria \\
\hline $\begin{array}{l}\text { T3: (a) Ampulla, duodenum: invading pancreas or retroperitoneum; } \\
\text { (b) jejunum, ileum: tumor invading subserosa }\end{array}$ & $\begin{array}{l}\text { T3: (a) Duodenum, ampulla, proximal jejunum: invading pancreas or retroperitoneum; } \\
\text { (b) distal jejunum and ileum: tumor invading subserosa }\end{array}$ \\
\hline T4: Tumor perforating serosa or invading adjacent structures & T4: Tumor invading peritoneum or other organs \\
\hline Large intestine & Large intestine \\
\hline Tx: Primary tumor cannot be assessed & Tx: Primary tumor cannot be assessed \\
\hline T0: No evidence of primary tumor & T0: No evidence of primary tumor \\
\hline $\mathrm{T} 1$ : Tumor $\leq 1 \mathrm{~cm}$ invading lamina propria or submucosa & T1: Tumor invading mucosa or submucosa: T1a: $<1 \mathrm{~cm}$; T1b: $1-2 \mathrm{~cm}$ \\
\hline $\mathrm{T} 2$ : Tumor $>1 \mathrm{~cm}$ or invading muscularispropria & $\mathrm{T} 2$ : Tumor $>2 \mathrm{~cm}$ or invading muscularispropria \\
\hline T3: Tumor invading subserosa & T3: Tumor invading subserosa or pericolonic/perirectal fat \\
\hline T4: Tumor perforating serosa or invading adjacent structures & T4: Tumor perforating visceral peritoneum or invading other organs or structures \\
\hline Appendiceal carcinoid & Large intestine \\
\hline Stage I T1N0M0 & Stage IA T1aNOM0 \\
\hline Stage II T2,T3N0M0 & Stage IB T1bN0M0 \\
\hline Stage III T4N0M0 & Stage IIA T2NOMO \\
\hline Stage III T1-4N1M0 & Stage IIB T3NOM0 \\
\hline Stage IV Any TNM1 & Stage IIIA T4N0M0 \\
\hline Carcinoid at other sites in the GI tract & Stage IIIB T1-4N1M0 \\
\hline Stage I T1N0M0 & Stage IV Any TNM1 \\
\hline Stage IIA T2NOM0 & Other sites \\
\hline Stage IIB T3NOM0 & Stage I T1N0M0 \\
\hline Stage IIIA T4N0M0 & Stage IIA T2NOMO \\
\hline Stage IIIB T1-4N1M0 & Stage IIB T3NOM0 \\
\hline Stage IV Any TNM1 & Stage IIIA T4N0M0 \\
\hline Small/large-cell enteric NECs: stage as carcinomas & Stage IIIB T1-4N1M0 \\
\hline Pancreatic/pulmonary NETs: stage as carcinomas & Stage IV Any TNM1 \\
\hline
\end{tabular}

AJCC American Joint Committee on Cancer, ENETS European Neuroendocrine Tumor Society, GI gastrointestinal, NEC neuroendocrine carcinoma, NET neuroendocrine tumor, SMA superior mesenteric artery 
in non-functioning tumors. However, data in this regard are not robust and need formal validation. Prophylactic cholecystectomy to prevent cholelithiasis is recommended in patients undergoing surgery if treatment with somatostatin analogues (SSA) is anticipated. Surgery of the primary tumor is often recommended in patients with metastatic small-bowel NETs to avoid bowel obstruction due to the neoplasm or to the fibrotic reaction commonly observed in the adjacent mesentery. Perioperative prophylactic therapy with SSA is mandatory in functional tumors to prevent carcinoid crisis. Finally, liver transplantation is controversial but can be considered in selected young patients with well-differentiated liver-only metastases, resected primary tumor and low Ki-67 index $(<5 \%)$, after a prolonged surveillance period to exclude rapidly progressing tumors. It may also be considered in patients with liver-only disease and a life-threatening functional syndrome.

Interventional radiology

In patients who are not suitable candidates for surgery, regional control of liver metastases may be achieved by different ablative techniques such as radiofrequency or laser ablation, and cryotherapy, among others [3, 4, 10]. Reduction of tumor burden often leads to a reduction of hormone secretion and, consequently, an improvement in symptom control. Other locoregional approaches include embolization of the hepatic artery by particles or cytotoxic agents (trans-arterial chemoembolization, TACE) [11]. The dual blood supply of the liver, with differential perfusion of metastatic lesions and normal hepatocytes by the hepatic artery and portal vein, respectively, make these therapeutic strategies tolerable to the healthy liver and particularly toxic to the cancer cell. They are generally employed with palliative purposes in patients with slow growing G1-2 functional tumors refractory to medical therapy, but may also be useful to reduce tumor burden and control tumor progression in non-functioning tumors. Doxorubicin, streptozocin, mitomycin and fluorouracil are commonly used agents in this context, although adequately sized randomized studies that properly evaluate the benefit-risk ratio of chemoembolization with that of mechanical embolization are lacking [12]. Clinical responses have been reported in up to $80 \%$ of the patients, and radiological responses in about $50 \%$. Common adverse events include pain, fever or elevation of liver enzymes. Severe complications occur in $10 \%$ of cases and include acute liver or renal failure, liver abscess, cholecystitis or carcinoid crisis. Radioembolization is an alternative technique of liverdirected therapy that is rapidly gaining acceptance and involves the percutaneous transarterial injection of micronsized embolic particles loaded with a radioisotope (most commonly yttrium-90), but well-designed, prospective randomized trials comparing it to the other modalities are lacking. Complications include nausea, fatigue, abdominal pain, hepatic dysfunction, vascular or biliary injury, fibrosis, radiation pneumonitis and gastrointestinal ulcers. However, the risk of complications may be minimized by meticulous pretreatment assessment, careful patient selection, and adequate dosimetry. Embolization is contraindicated in patients with portal-vein thrombosis, liver insufficiency, biliary obstruction or prior Whipple procedure.

\section{Systemic therapy}

Patients with advanced disease have limited therapeutic options. Indeed, NETs exhibit low susceptibility to conventional cytotoxic therapy. Long-acting SSAs are currently the best option to provide symptomatic relief of hormonal syndrome, and increasing clinical data indicate that they also retard disease progression [13, 14]. Symptomatic therapy in functional NETs may also include other specific drugs depending upon the hormone or peptide secreted in excess by the tumor, such as proton pump inhibitors in gastrinomas, diazoxide or glucagon in insulinomas, insulin or other pancreatic hormones in somatostatinomas, etc. Other options to achieve disease control include peptide-receptor radionuclide therapy, although availability is still limited in many countries [15]. Randomized trials are currently ongoing, the results of which shall contribute to properly assess the benefit-risk balance of this promising approach. Chemotherapy may be considered in certain disease settings, depending upon tumor differentiation, bulk and kinetics, particularly in those of pancreatic origin [16-18]. Finally, two targeted agents, sunitinib and everolimus, have demonstrated in randomized placebo-controlled trials a clinically relevant antiproliferative effect in well-differentiated NETs of pancreatic origin [19, 20]. Everolimus may also prolong progressionfree survival in non-pancreatic NETs [21]. However, the magnitude of this effect is more limited. A number of other targeted agents are currently in clinical development and may add to the treatment armamentarium in the near future. A therapeutic algorithm is proposed in Figs. 1, 2 and 3.

\section{Somatostatin analogues and interferon}

SSA remain the most effective drugs for symptom control in functional GEP-NETs. Indeed, 70-80 \% of patients experience resolution of diarrhea or flushing, and about $40 \%$ achieve biochemical response, although tumor regression is rare. Nevertheless, two recent controlled trials indicate they do have antiproliferative activity. The first one, a randomized double-blind placebo-controlled trial 


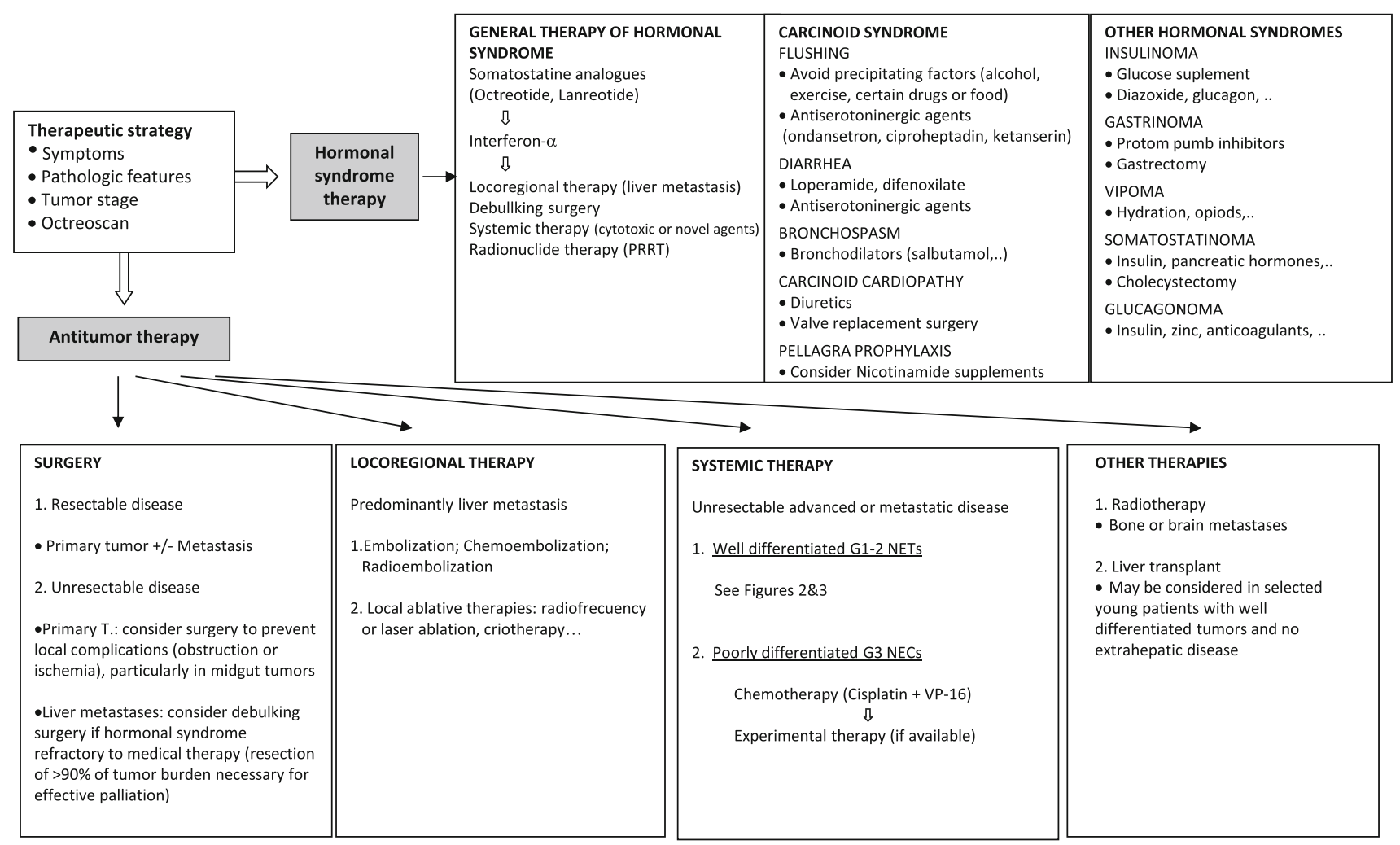

Fig. 1 Therapeutic algorithm in GEP-NENs

conducted in 85 patients with well-differentiated G1 metastatic midgut NETs, showed that patients treated with octreotide long-acting repeatable (LAR) (30 mg every 28 days) had a significantly longer time to tumor progression (14.3 months) than patients treated with placebo (6 months) (HR 0.34, $95 \%$ CI 0.20-0.59, $P<0.001$ ). The greatest effect was observed in patients with low hepatic tumor load and resected primary tumor [13]. The most recent survival update of this trial did not show, however, any difference in overall survival among study arms. Further evidence of the antiproliferative effect of SSA has been recently provided by the CLARINET study [14]. This phase III, double-blind, placebo-controlled trial included 204 patients with well or moderately differentiated (Ki-67 $<10 \%$ ) non-functioning GEP-NETs (45\% pancreatic, $36 \%$ midgut, $7 \%$ hindgut and $13 \%$ of unknown primary), that were randomly allocated to receive prolonged release lanreotide (lanreotide autogel $120 \mathrm{mg}$ ) or placebo every 4 weeks. Treatment with lanreotide significantly prolonged progression-free survival (PFS) over placebo (median not reached with lanreotide versus 18 months with placebo, HR 0.47, $P<0.001)$. Of note, one-third of enrolled patients had over $25 \%$ liver involvement, and as opposed to that observed in the PROMID study, patients with high hepatic tumor load also benefited from lanreotide therapy. The most convenient formulations are lanreotide autogel $(60,90$ or $120 \mathrm{mg})$ and octreotide LAR $(10,20$ or $30 \mathrm{mg})$.
Currently recommended antiproliferative doses are octreotide LAR $30 \mathrm{mg}$ or lanreotide autogel $120 \mathrm{mg}$ every 28 days. When used for syndrome control starting doses can be lower and then clinically monitored. Doses above $120 \mathrm{mg}$ for Lanreotide autogel or $30 \mathrm{mg}$ for Octreotide LAR are off-label but can sometimes be needed in selected cases. Short-acting subcutaneous octreotide is still necessary to be used in combination with depot formulations during the first 2 weeks of therapy until adequate plasma therapeutic levels are achieved, and for rescue treatment in carcinoid syndrome exacerbations. Adverse effects of SSA are mild and manageable, and include malabsorption, endocrine disturbances (hypothyroidism, hypoglycemia, or, more commonly, hyperglycemia), pain and erithema at the site of injection, hypersensitivity reactions and cholelithiasis on long-term use [3, 4].

Interferon is also effective in terms of symptomatic control of the hormonal syndrome, but its use is limited by substantial adverse effects (alopecia, anorexia, fatigue, depression, liver toxicity, weight loss, fever, a flu-like syndrome and myelosuppression). Although some small randomized trials have shown a trend towards an improved disease progression or overall survival when interferon was added to SSA as first line treatment, this benefit was not consistently observed in all trials or did not reach statistical significance $[3,4]$. For this reason it is generally indicated after failure of other therapies. 


\section{A Asymptomatic disease}

If complete resection possible $\longrightarrow$ Resection of metastases \pm primary tumor

Unresectable G1 disease $\longrightarrow$ Observe with CT scan/3-6 m vs SSA* $\longrightarrow$\begin{tabular}{l|}
$\begin{array}{l}\text { If clinically significant } \\
\text { disease progression } \\
\text { see below }\end{array}$ \\
\hline
\end{tabular}

* Somatostatin analogues may be considered if positive octreoscan (octreotide for midgut; lanreotide for enteric, lung or pancreatic primaries)

\section{B Symptomatic patients or G2 or Significant tumor burden or Disease progression}

If complete resection possible $\longrightarrow$ Resection of metastases \pm primary tumor

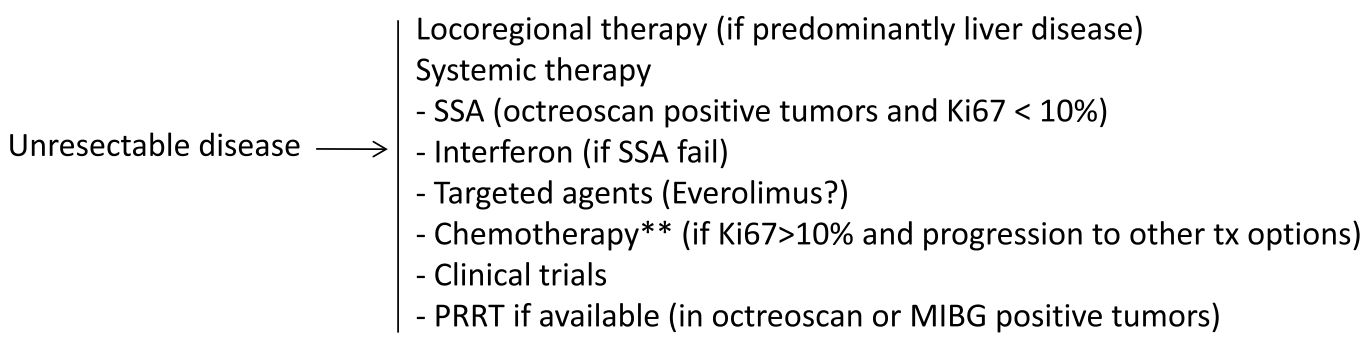

** Options: Streptozotocin + 5-Fluorouracil

Fig. 2 Management of advanced/metastatic G1-G2 enteric NETs

\section{A Asymptomatic disease}

If complete resection possible $\longrightarrow$ Resection of metastases \pm primary tumor

Unresectable G1 disease $\longrightarrow$ Somatostatin analogues* if octresocan $+\longrightarrow$ If clinically significant disease progression see below

* Lanreotide preferable

\section{B Symptomatic patients or G2 or Significant tumor burden or Disease progression}

If complete resection possible $\longrightarrow$ Resection of metastasis \pm primary tumor

Unresectable disease $\longrightarrow \mid \begin{aligned} & \text { Locoregional therapy (if predominantly liver disease) } \\ & \text { Systemic therapy } \\ & \text { - Lanreotide (octreoscan positive tumors and Ki67 < 10\%) } \\ & \text { - Targeted agents (Everolimus or Sunitinib) } \\ & \text { - Chemotherapy** } \\ & \text { - Clinical trials } \\ & \text { - PRRT if available (in octreoscan or MIBG positive tumors) }\end{aligned}$

** Options: Streptozotocin + 5-Fluorouracil or Doxorubicin, Temozolomide + Capecitabine

Fig. 3 Management of advanced/metastatic G1-G2 pancreatic NETs 
Peptide-receptor radionuclide therapy

Patients with advanced disease and a positive octreoscan may be considered for peptide receptor radionuclide therapy (PRRT). PRRT primarily utilizes one of two radioisotopes, 90 Yttrium (90Y) or 177 Lutetium (177Lu), attached to a SSA via the chelating agent 1,4,7,10-tetraazacyclo-dodecane-1,4,7,10-tetraacetic acid (DOTA). 90 Yttrium may be delivered as (90Y-DOTA0, Tyr3) Octreotide (90Y-DOTATOC) or as the newer (90Y-DOTA0, Tyr3) Octreotate (90Y-DOTATATE). 90 Yttrium-labeled SSAs have been reported to induce not only tumor stabilization but also tumor regression (objective partial responses) in up to $30 \%$ of GEP-NETs [15]. However, experience is limited to single institution selected series and randomized controlled trials are currently ongoing and shall provide more solid evidence regarding safety and efficacy of this treatment modality. In addition, the nonavailability of this therapeutic strategy in most countries further limits its widespread use. The appropriate timing of this therapeutic intervention or the relative long-term benefit-risk ratio compared to other treatment options are key questions that remain unanswered. Alternatively, 131 Iodine-metaiodiobenzylguanidine (MIBG) therapy may be considered in advanced tumors with a positive MIBG scan (20-50\% of NETs).

\section{Chemotherapy}

Conventional cytotoxic therapy is considered as the first treatment option for poorly differentiated or rapidly progressive advanced GEP-NETs. The combination of cisplatin and etoposide is the most widely used chemotherapy regimen in this setting, and is associated with a relatively high (27-54\%) albeit short-lived response rate and a poor survival (15-19 months). Well-differentiated tumors, however, have a more indolent clinical course and exhibit poor sensitivity to chemotherapy. Among them, radiological, biochemical and clinical responses are more commonly encountered in pancreatic NETs (pNETs) than in enteric or non-pancreatic NETs. For this reason, chemotherapy is considered an early therapeutic intervention in advanced NETs of pancreatic origin only. In contrast, chemotherapy plays a minor role in the management of gastrointestinal NETs, being generally reserved for patients with tumors refractory or progressive to other therapeutic strategies (SSA, interferon, locoregional ablative therapy, PRRT, etc.) and may likely soon be replaced by new emergent targeted agents.

The most commonly employed regimens generally combine streptozotocin with doxorubicin or 5-fluorouracil, with response rates of $8-20 \%$ reported in recent series $[3,4]$. These combination schedules showed improved response rates and/or survival compared to single agent streptozotocin in some old classical randomized clinical trials [16-18]. However, these studies are methodologically weak for current standards (small sample size, crucial baseline clinical data not reported, lack of uniformity in the assessment of tumor response), and antitumor efficacy of these agents against placebo or best supportive care has never been adequately evaluated in controlled trials. Other active drugs in NETs include dacarbazine or temozolomide. Temozolomide seems particularly active in $\mathrm{O}^{6}$ methyl-guanine-methyl-transferase (MGMT)-deficient tumors, an enzyme involved in the repair of temozolomideinduced DNA damage. The combination of temozolomide with capecitabine is a convenient and widely used oral drug regimen, with a favorable toxicity profile and promising results from small heterogeneous series [22]. However, the safety and efficacy of these regimens have never been properly assessed against other therapeutic options in this context.

\section{Novel targeted agents}

Several recent adequately powered randomized trials have for the first time demonstrated that there are agents able to positively impact the outcome of this disease, in particular, angiogenesis and mTOR inhibitors. Sunitinib, a tyrosine kinase inhibitor that targets, among others, VEGFR, PDGFR and c-kit, was evaluated in a randomized placebocontrolled trial in patients with progressive well-differentiated pancreatic NETs not suitable for curative surgery [19]. Crossover of placebo patients to sunitinib at disease progression was not initially permitted. About two-thirds of patients had received prior chemotherapy and 20-22\% were receiving concomitant SSA. This study aimed to include 340 patients but was prematurely closed with 171 patients due to the excess of deaths observed in the placebo arm. At that point, patients who had progressed on the placebo arm became candidates for open-label sunitinib therapy. Although objective responses were only observed in $9 \%$ of treated patients, PFS was significantly superior in patients treated with sunitinib versus those receiving placebo (11.4 versus 5.5 months; HR $0.42 ; P=0.001$ ), as well as overall survival (HR $0.41, P=0.02$ ) in the first interim analysis. With further follow-up, and after $69 \%$ of patients had crossed over to sunitinib therapy, overall survival still favored the sunitinib arm (30.5 versus 24.4 months), although this difference was no longer statistically significant (HR 0.74, $P=0.19$ ).Common side effects of drug included nausea, vomiting, asthenia and hair-color changes, which were generally manageable, and quality of life was not adversely affected by sunitinib therapy. The main severe adverse events (grade 3-4) were 
neutropenia, hypertension and hand-foot syndrome, with incidence rates of $6-12 \%$.

Regarding mTOR inhibitors, two large phase III trials have explored safety and efficacy of everolimus in different NET settings. The first one, the RADIANT-3 study, was conducted in 410 patients with progressive advanced low or intermediate grade pancreatic NETs who were randomly assigned to everolimus or placebo with a double-blind crossover study design [20]. Fifty percent of patients had received prior chemotherapy. Again, objective responses were low $(5 \%)$, but patients treated with everolimus had a significantly longer PFS than those treated with placebo (11.4 versus 5.4 months, HR $0.34, P<0.0001$ ). No impact was observed on survival, likely due to the fact that $73 \%$ of patients of the placebo arm crossed over to everolimus upon disease progression. The safety profile of everolimus was acceptable, being stomatitis, rash, diarrhea, fatigue, and infections the most common side effects. About 5-7\% of patients developed grade 3-4 toxicities, such as stomatitis, anemia and hyperglycemia. Pneumonitis and interstitial lung disease $(17 \%)$ represent the most important clinical concerns, although they were severe (grade 3-4) in only $2 \%$ of treated patients. In addition to its antiproliferative effect, everolimus has proven effective in controlling refractory insulinoma syndrome. The second large study, the RADIANT-2, included 429 patients with low or intermediate grade advanced NETs with a history of carcinoid syndrome (the great majority of non-pancreatic origin). These patients were randomly allocated to receive octreotide LAR with placebo or with everolimus, with cross-over to everolimus allowed at disease progression for placebo allocated patients [21]. Median PFS evaluated by local investigators was 12 versus 8.6 months for everolimus versus placebo treated patients, respectively (HR 0.78 , $95 \%$ CI $0.62-0.98, P_{\text {(one-tailed) }}=0.036$ ). Independent central review documented a PFS improvement favoring everolimus treatment of similar magnitude (16.4 versus 11.3 months; HR $0.77,95 \%$ CI $0.59-1.00 ; P_{\text {(one-tailed) }}$ $=0.026)$, but the observed difference missed the prespecified significance boundary $(P \leq 0.0246)$. Similarly to that observed in the prior study, a high proportion of patients on the placebo arm crossed over to everolimus at disease progression $(58 \%)$ and no differences were observed in overall survival among study arms. Based on these results, regulatory agencies granted marketing authorization to sunitinib (2010) and everolimus (2011) for the treatment of progressive advanced PNETs, but everolimus was not approved for the treatment of NETs of nonpancreatic origin. Another agent in advanced clinical evaluation is bevacizumab, which is currently being tested in a randomized trial against PEG-interferon in carcinoid tumors, the results of which are eagerly awaited in the near future [23].
In conclusion, sunitinib and everolimus are new treatment options in patients with low or intermediate grade pancreatic NETs in whom disease progression has been documented. Whether these agents should be employed before or after chemotherapy failure is a matter of debate. The efficacy of both agents seems similar, although no formal head-to-head comparisons exist nor are they expected to be performed in the near future. Regarding the use of everolimus in non-pancreatic functional NETs, no firm recommendations can be made, as the reported benefit is of lower magnitude and regulatory approval has not been granted, within a clinical scenario with long survival and scant therapeutic options. Efforts to provide predictive biomarkers to help select subgroups of patients that are more likely to benefit from each individual drug are certainly warranted.

\section{Follow-up}

Follow-up recommendations are based on expert opinion as there is no solid evidence to support the type and frequency of performance of specific procedures. Patients with localized G1-2 NETs who have undergone complete resection are recommended to be followed every 6-12 months the first 3 years, and then annually, at least up to the 5th year following surgery, in order to detect potentially resectable recurrences. Follow-up intervals shall be shorter in patients with G3 NECs (every 3-6 months during the first 3 years). General tumor markers (chromogranin A in G1-2 NETs or neuron-specific enolase in G3 NECs) and imaging procedures (CT scan or MRI) shall be performed in these follow-up visits. The role of octreoscan in this setting is controversial in the absence of clinical, biochemical or radiological suspicion of relapse. Specific markers (urinary 5-HIAA acid for midgut NETs; insulin, gastrin or others for specific functioning pNETs) may be also performed in tumors that presented a hormonal syndrome or if clinically indicated.

Follow-up of patients with advanced disease shall be customized depending upon tumor kinetics (Ki-67 proliferative index and actual growth rate documented by serial CT scans), treatment strategy, side effects of therapy and general health condition. Visits may be done every 6 months for G1, slow growing tumors, in clinically stable patients under low-toxic therapies (i.e., SSA). These intervals, however, shall be scheduled far more frequently for those with faster tumor kinetics and/or receiving more toxic agents. In this last scenario, imaging procedures are recommended to be performed every 3-6 months.

Conflict of interest None. 


\section{References}

1. Lawrence B, Gustafsson BI, Chan A, Svejda B, Kidd M, Modlin IM. The epidemiology of gastroenteropancreatic neuroendocrine tumors. Endocrinol Metab Clin N Am. 2011;40:1-18.

2. Garcia-Carbonero R, Capdevila J, Crespo-Herrero G, Díaz-Pérez JA, Martínez Del Prado MP, Alonso Orduña V, et al. Incidence, patterns of care and prognostic factors for outcome of gastroenteropancreatic neuroendocrine tumors (GEP-NETs): results from the National Cancer Registry of Spain (RGETNE). Ann Oncol. 2010;21:1794-803.

3. Modlin IM, Oberg K, Chung DC, Jensen RT, de Herder WW, Thakker RV, et al. Gastroenteropancreatic neuroendocrine tumours. Lancet Oncol. 2008;9:61-72.

4. Garcia-Carbonero R, Sevilla I, Aller J, Martin E (eds). Manual de Diagnóstico y Tratamiento de los Tumores Neuroendocrinos. In: Editorial TACTICS Medicina y Desarrollo S.L., $2^{\mathrm{a}}$ Edición (4 de Octubre de 2013) (ISBN 978-84-6958312-8). http://www.getne.org/ÁreadeProfesionales/Materialdetrabajoen TNEs.aspx

5. Rindi G, Arnold R, Bosman FT. Nomenclature and classification of neuroendocrine neoplasms of the digestive system. In: Bosman TF, Carneiro F, Hruban $\mathrm{RH}$, Theise ND, editors. WHO classification of tumours of the digestive system. 4th ed. Lyon: International Agency for Research on cancer (IARC); 2010. p. 13.

6. Rindi G, Klöppel G, Alhman H, Caplin M, Couvelard A, de Herder WW, et al TNM staging of foregut (neuro)endocrine tumors: a consensus proposal including a grading system. Virchows Arch. 2006;449:395-401.

7. Rindi G, Klöppel G, Couvelard A, Komminoth P, Körner M, Lopes JM, et al. TNM staging of midgut and hindgut (neuro) endocrine tumors: a consensus proposal including a grading system. Virchows Arch. 2007;451:757-62.

8. Sobin L, Gospodarowicz M, Wittekind C (eds). TNM classification of malignant tumours, 7th edn. Oxford: Wiley-Blackwell; 2010.

9. García-Carbonero R, Vilardell F, Jiménez-Fonseca P, González-Campora R, González E, Cuatrecasas M, et al. Guidelines for biomarker testing in gastroenteropancreatic neuroendocrine neoplasms: a national consensus of the Spanish Society of Pathology and the Spanish Society of Medical Oncology. Clin Transl Oncol. 2014;16(3):243-56.

10. Akyildiz HY, Mitchell J, Milas M, Siperstein A, Berber E. Laparoscopic radiofrequency thermal ablation of neuroendocrine hepatic metastases: longterm follow-up. Surgery. 2010;148(6):1288-93.

11. Yang TX, Chua TC, Morris DL. Radioembolization and chemoembolization for unresectable neuroendocrine liver metastases-a systematic review. Surg Oncol. 2012;21(4):299-308.
12. Mayo SC, de Jong MC, Bloomston M, Pulitano C, Clary BM, Reddy SK, et al. Surgery versus intra-arterial therapy for neuroendocrine liver metastasis: a multicenter international analysis. Ann Surg Oncol. 2011;18(13):3657-65.

13. Rinke A, Müller HH, Schade-Brittinger C, Klose KJ, Barth P, Wied M, et al. PROMID Study Group. Placebo-controlled, double-blind, prospective, randomized study on the effect of octreotide LAR in the control of tumor growth in patients with metastatic neuroendocrine midgut tumors: a report from the PROMID Study Group. J Clin Oncol. 2009;27:4656-63.

14. Caplin M, Ruszniewski P, Pavel M, Ćwikła JB, Phan A, Raderer M, et al. A randomized, double blind, placebo controlled study of lanreotide antiproliferative response in patients with gastroenteropancreatic neuroendocrine tumors (CLARINET). In Presented at ECC 2013, abstract LBA3.

15. Kwekkeboom DJ, de Herder WW, Kam BL, van Eijck CH, van Essen M, Kooij $\mathrm{PP}$, et al. Treatment with the radiolabeled somatostatin analog [177 Lu-DOTA 0 , Tyr3]octreotate: toxicity, efficacy, and survival. J Clin Oncol. 2008;26(13):2124-30.

16. Moertel CG, Hanley JA, Johnson LA. Streptozocin alone compared with streptozocin plus fluorouracil in the treatment of advanced islet-cell carcinoma. N Engl J Med. 1980;303(21):1189-94.

17. Moertel CG, Lefkopoulo M, Lipsitz S, Hahn RG, Klaassen D. Streptozocindoxorubicin, streptozocin-fluorouracil or chlorozotocin in the treatment of advanced islet-cell carcinoma. N Engl J Med. 1992;326:519.

18. Engstrom PF, Lavin PT, Moertel CG, Folsch E, Douglass HO Jr. Streptozocin plus fluorouracil versus doxorubicin therapy for metastatic carcinoid tumor. J Clin Oncol. 1984;2:1255.

19. Raymond E, Dahan L, Raoul JL, Bang YJ, Borbath I, Lombard-Bohas C, et al. Sunitinib malate for the treatment of pancreatic neuroendocrine tumors. N Engl J Med. 2011;364:501-13.

20. Yao JC, Shah MH, Ito T, Bohas CL, Wolin EM, Van Cutsem E, et al. Everolimus for advanced pancreatic neuroendocrine tumors; RAD001 in Advanced Neuroendocrine Tumors, third trial (RADIANT-3) Study Group. N Engl J Med. 2011;364:514-23.

21. Pavel ME, Hainsworth JD, Baudin E, Peeters M, Hörsch D, Winkler RE, et al. RADIANT-2 Study Group. Everolimus plus octreotide long-acting repeatable for the treatment of advanced neuroendocrine tumours associated with carcinoid syndrome (RADIANT-2): a randomised, placebo-controlled, phase 3 study. Lancet. 2011:378(9808):2005-12.

22. Strosberg JR, Fine RL, Choi J, Nasir A, Coppola D, Chen DT, et al. First-line chemotherapy with capecitabine and temozolomide in patients with metastatic pancreatic endocrine carcinomas. Cancer. 2011;117(2):268-75.

23. Benavent M, de Miguel MJ, Garcia-Carbonero R. New targeted agents in gastroenteropancreatic neuroendocrine tumors. Target Oncol. 2012;7(2):99-106. 